\title{
Moderne empirische (Nicht-)Kundenforschung
}

\author{
Plädoyer für einen Schritt zurück - Teil 1
}

\author{
Tobias Schütz und Marko Sarstedt
}



Prof. Dr. Tobias Schütz ist Professor für Marketing und Customer Science an der ESB Business School der Hochschule Reutlingen. Bevorzugte Forschungsgebiete: Kundenzufriedenheit und Kundenbindung, Services Marketing, Employer Branding.

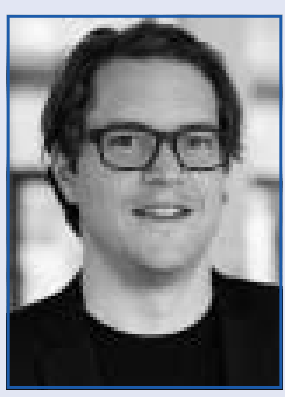

Prof. Dr. Marko Sarstedt ist Inhaber des Lehrstuhls für Marketing an der Ottovon-Guericke-Universität Magdeburg und Conjoint Professor an der University of Newcastle, Australien. Bevorzugte Forschungsgebiete: Forschungsmethoden, Datenqualität, Konsumentenverhalten.

Kundenforschungsprojekte sind häufig durch einen beschränkten Fokus auf bestimmte Untersuchungsobjekte, Forschungsdesigns und Datenanalyseverfahren geprägt. Leider ist das häufig zu beobachtende Standardvorgehen nicht immer korrekt und liefert in vielen Fällen sogar fehlerhafte Ergebnisse. Die Diskussion des optimalen Untersuchungsobjekts und des geeigneten Untersuchungsdesigns sind Gegenstand des ersten Teils dieses Beitrages. Teil 2 im nächsten WiStHeft, Nr. 5/2016, S. 224 ff. ist der Datenanalyse und der Synthese der Erkenntnisse gewidmet.

Stichwörter: Kundenforschung, Forschungsobjekt, Forschungsdesign, Datenerhebung, Datenanalyse

\section{Ausgangslage}

Projekte der Kundenforschung, egal ob es sich um Studierendenprojekte, Praktikerstudien oder wissenschaftliche Beiträge handelt, lassen sich auf Basis von drei Dimensionen charakterisieren: Das Objekt der Forschung, die Form des Forschungsdesigns und die Methode der Datenanalyse. Das Objekt der Forschung beschreibt, wer oder was im Rahmen des Forschungsprojekts untersucht werden soll. Beispielsweise könnte der Fokus auf der Untersuchung von Bestandskunden oder einer bestimmten Kun- dengruppe liegen. Das Forschungsdesign legt fest, wie eine Forschungsfrage untersucht werden soll. Je nachdem, wie hoch der Strukturierungsgrad der Fragestellung ist, unterscheidet man zwischen explorativen, deskriptiven und kausalen Designs (vgl. Sarstedt/Mooi, 2014, S. 13 f.). Zuletzt muss entschieden werden, welche Datenanalysemethode verwendet werden soll. Hier reicht das Spektrum von einfachen deskriptiven Analysen (Berechnung von Lage- und Streumaßen) bis hin zu teilweise sehr komplexen multivariaten Analyseverfahren wie der Strukturgleichungsmodellierung.

Wie in vielen anderen Feldern der wissenschaftlichen und praktischen Betriebswirtschaft gibt es auch in der Kundenforschung ein Verständnis über die typische Ausgestaltung dieser drei Dimensionen zur Beantwortung bestimmter Fragestellungen. Leider sind die üblichen Standardvorgehen nicht immer optimal. So wählen Studierende der Betriebswirtschaft in Abschlussarbeiten häufig hoch strukturierte Interviewmethoden für diffuse Forschungsfragen, deren Beantwortung eigentlich nach einem wenig strukturierten, qualitativen Design verlangt. In Unternehmen kommen bei der Steuerung und Incentivierung von kundennahen organisatorischen Einheiten Leistungskennzahlen auf Basis von Mittelwerten zum Einsatz. Sensitivitätsanalysen, Signifikanztests oder multivariate Analysen zur Erklärung der Zusammenhäng bleiben häufig aus. Zuletzt ist in wissenschaftlichen Beiträgen des modernen Marketings die hohe Komplexität der angewendeten Methode häufig entscheidender als deren inhaltliche Sinnhaftigkeit oder das Ergebnis der Analyse. Dies zeigt sich an den teilweise trivialen Ergebnissen, die mit großem Analyseaufwand produziert wurden und in führenden Fachzeitschriften dokumentiert sind.

In Anbetracht dieses Status Quo plädieren wir bei der Konzipierung von Kundenforschungsprojekten für einen „Schritt zurück“ im doppelten Sinne. Einerseits erlaubt ein Schritt zurück das große Ganze, also den gesamten Baukasten der Kundenforschung zu betrachten und den für die konkrete Forschungsfrage optimalen Baustein auszuwählen. Um ein Verständnis für die Vielzahl der Möglichkeiten in den einzelnen Dimensionen zu schaffen, betrachten wir die drei Dimensionen der Kundenanalyse zunächst isoliert. Gegenstand des ersten Teils des Beitrages sind das Forschungsobjekt und das Forschungsdesign. Im zweiten Teil erläutern wir die Kriterien zur Auswahl und Anwendung der Methode der Datenanalyse und betrachten die Synthese der Ergebnisse auf deren Basis wir Empfehlungen für die Kundenforschung ableiten. 


\section{Objekt der Forschung}

Der überwiegende Teil wissenschaftlicher und praktischer Studien zur Analyse von Kundenbedürfnissen, sowie zur Bewertung und Verbesserung von Kundenzufriedenheit, Kundenbindung und Loyalität basiert auf Feedback der aktuell bestehenden Kundenstruktur eines Unternehmens oder einer Marke. Insbesondere bei unternehmensübergreifenden Benchmarking-Studien stehen die Zufriedenheitsurteile der Bestandskunden im Fokus der Betrachtung und werden regelmäßig aufwändig vermarktet. In Deutschland küren zum Beispiel DIE WELT und die Service Value $\mathrm{GmbH}$ jährlich die Sieger der branchenübergreifenden Service-Champions Studie, in deren Rahmen 1.588 Unternehmen auf Basis von ca. 1 Million Kundenurteilen bewertet und nach ihren Ergebnissen gelistet und prämiert werden (vgl. DIE WELT, 2014). Der quartalsweise veröffentlichte American Customer Satisfaction Index (ACSI) ist in den USA ein wichtiger Gradmesser für das kundengerichtete Leistungsprofil von Unternehmen, Branchen und Wirtschaftssektoren (vgl. ACSI, 2015). In der Automobilbranche warten Entscheider jedes Jahr weltweit auf die Ergebnisse der Kundenzufriedenheitsstudien von J.D. Power \& Associates in den Disziplinen Kurzzeitqualität (Initial Quality Survey), Langzeitqualität (Vehicle Dependability Study), Designqualität (Automotive Performance Execution and Layout), Vertriebszufriedenheit (Sales Satisfaciton Index) und Servicequalität (Customer Service Index) (vgl. JDPA, 2015).

Die Ergebnisse dieser Studien sind bereits seit Jahrzehnten routinemäßig in die Anreizstrukturen der Unternehmen eingebunden und beeinflussen unternehmerische Entscheidungen in den Bereichen Produktentwicklung, Vertrieb, Serviceorganisation und Personal (vgl. Hauser et. al, 1990, S. 327 f., oder Sharma/Sarel, 1995, S. 17 ff.). Die empirische Relevanz von Kundenmeinungen für unternehmerische Entscheidungen ist als Indikator für ein reales Bestreben zu kundenorientiertem Denken und Handeln interpretierbar und damit im ersten Schritt positiv zu bewerten. Die Betrachtung der absoluten Ergebnisse der Kundenfeedbacks lässt aber Zweifel an der Sinnhaftigkeit dieser Praxis aufkommen.

Regelmäßig sind die Kundenfeedbacks am oberen Rand der verwendeten Zufriedenheitsskalen konzentriert und erlauben keine systematische Differenzierung zwischen den bewerteten Marken, Produkten oder Dienstleistungen. Ein Beispiel: In der J.D. Power \& Associates Automotive Kundenzufriedenheitsstudie 2014 Germany Vehicle Ownership Satisfaction Study erzielte die erstplatzierte Marke Porsche 835, die auf Rang 24 gelistete Marke Dacia 756 Indexpunkte (vgl. J.D. Power, 2014). 16 der 26 gelisteten Marken verteilten sich auf Bewertungen von 772 bis 800. Da die Indexskala die lineare Hochrechnung einer 10Punkte Bewertungsskala ist, unterscheiden sich also über $60 \%$ der Marken um weniger als 0,3 Skalenpunkte. Statistisch signifikante Unterschiede zwischen benachbarten Marken sind aufgrund von Ceiling-Effekten (vgl. Uttl, 2005) in den wenigsten Fällen nachweisbar.
Aus Vermarktungsgesichtspunkten ist die Top-Platzierung in einer bekannten Kundenzufriedenheitsstudie wertvoll. Als Informationsbasis für die Verbesserung des eigenen Leistungsportfolios sind diese Studien aber unzureichend. Ihre größte Schwäche liegt in der Stichprobe, die definitionsgemäß aus den aktuellen Kunden des Unternehmens besteht. Da dieser Personenkreis die Produkte und Dienstleistungen des bewerteten Unternehmens zumindest als so attraktiv einstuft, dass er sich zu deren Konsum entschieden hat, sind die guten und wenig differenzierten Zufriedenheitsbewertungen nur schlüssig. Potenzielle Kunden, welche die Leistungen des Unternehmens als unattraktiv bewerten und darum nicht zum Kundenstamm gehören, werden systematisch von der Bewertung ausgeschlossen. Es liegt folglich ein systematischer Stichprobenbias vor (vgl. Shiu/Hair/Bush/Ortinau, 2009) und das Feedback ist kein repräsentatives Abbild der aggregierten Konsumentenmeinung.

In der Praxis entsteht der Eindruck, dass im Streben nach stets höheren Kundenzufriedenheitswerten bei manchen Entscheidern eine kritische Frage in den Hintergrund getreten ist: Wie relevant ist eine weitere Steigerung der Kundenzufriedenheit für das Geschäftsmodell? Vor dem Hintergrund dieser Frage sind die Feedbacks der potenziellen Kunden für unternehmerische Entscheidungen meist relevanter als die der Bestandskunden. Selbstverständlich sind nicht alle Nicht-Kunden relevante Informationsquellen. Wenn geografische, zeitliche, budgetäre, etc. Restriktionen einzelne Marktsegmente ex-ante vom Konsum ausschließen, ist eine intensive Auseinandersetzung nicht zielführend. Allerdings können vier Typen von NichtKunden unterschieden werden, die meist von hoher Relevanz sind: Avoider, Rejecter, Shopper und Defecter (Schütz/Kindermann, 2012, S. 103; vgl. Abb. 1). Avoider sind Konsumenten, die den über psychographische, behavioristische oder demografische Kriterien (vgl. Jobber, 2012, S. 122 ff.) definierten Zielsegmenten des Unternehmens angehören, dessen Produkte und Dienstleistungen aber nicht in ihr relevantes Entscheidungsset aufgenommen haben. Bei Kaufentscheidungen werden Leistungen systematisch ignoriert bzw. aktiv vermieden.

Die zweite Gruppe bilden potenzielle Kunden, die Leistungen des Unternehmens in Erwägung gezogen und sich evtl. sogar ein Angebot eingeholt, sich aber final für den Kauf eines Konkurrenzproduktes entschieden haben. Da das Leistungsangebot die Konsumenten also im ersten $\mathrm{Zu}-$ griff bereits angesprochen hat, im Vergleich aber zurückgewiesen wurde, wird diese Gruppe Rejecter genannt.

Konsumenten, welche die Leistung des Unternehmens im Auswahlprozess in Erwägung gezogen, den Kaufprozess aber noch nicht final abgeschlossen haben, werden als Shopper bezeichnet. Im Konsumgüterbereich variiert die Zeitspanne zwischen der ersten Informationssuche und der finalen Kaufentscheidung deutlich. Bei Gütern des alltäglichen Bedarfs werden ca. $75 \%$ der Kaufentscheidungen am Point of Sale getroffen werden (vgl. Rettie/Brewer, 2000, S. 56) und Informationsaufnahme und Entscheidung 
fallen faktisch zusammen. Folglich ist für diese Gütertypen die Gruppe der Shopper weitestgehend irrelevant. Bei Gütern des Such- und Vergleichskaufs wie beispielsweise Markenbekleidung oder Unterhaltungselektronik liegen aber häufig Zeiträume von mehreren Wochen und Monaten zwischen der ersten Informationsaufnahme und der finalen Kaufentscheidung. Dies gilt ebenso für Sonderprodukte wie zum Beispiel Luxusuhren, Premiumfahrzeuge und teure Weine (vgl. Nieschlag/Dichtl/Hörschgen, 2002, S. 581 f.). Für diese Gütertypen stellen Shopper einen relevanten Personenkreis dar, der aktiv durch Marktforschungsmaßnahmen adressiert werden sollte.

Defecter sind ehemalige Kunden eines Unternehmens. Sie haben die Geschäftsbeziehung beendet und aufgehört, dessen Leistungen zu kaufen und zu konsumieren.

Die Erforschung der Motivationen dieser vier Typen von Nicht-Kunden ist für die Geschäftsentwicklung eines Unternehmens von hoher Relevanz (vgl. Abb. 1). Das Verständnis des Vermeidungsverhaltens der Avoider gibt unmittelbaren Aufschluss über Möglichkeiten zur Steigerung der Zahl faktisch adressierbarer Konsumenten. Eine Verringerung der Rejecter resultiert in einer besseren Abschlussquote und damit in einer höheren Kundenzahl. Gelingt die Identifikation und Befragung von Shoppern, so erlaubt dies nicht nur Rückschlüsse auf mögliche Maßnahmen zur Verkürzung des Kaufentscheidungsprozesses, sondern bietet die Möglichkeit, Marktforschungs- und Vertriebsaktivitäten zu verbinden und direkte Abschlüsse zu erzielen. Werden die Abwanderungsgründe der Defecter verstanden, so können zukünftig Schlüsselereignisse vermieden werden, die in der Vergangenheit die Abwanderung von Bestandskunden ausgelöst haben. Aktivitäten, die auf die Ergründung der Einstellungen und des Verhaltens dieser Gruppen von Nicht-Kunden ausgerichtet sind, haben folglich unmittelbaren Einfluss auf den zukünftigen Vertriebserfolg. Darum sollte ihnen bei der Planung der Marktforschungsaktivitäten eine hohe Bedeutung beigemessen werden.

Im Gegensatz dazu können die Ermittlung der Zufriedenheit der Bestandskunden und die Ableitung von Aktivitäten zur Verbesserung dieser den Markterfolg primär durch eine verbesserte Weiterempfehlungsrate und somit nur mittelbar beeinflussen. In der Literatur werden mit einer hohen Kundenzufriedenheit zudem niedrigere Kosten der Kundenakquise, eine höhere Auswahl-, eine geringere Abwanderungswahrscheinlichkeit und damit ein höherer Unternehmenswert assoziiert (vgl. Denove/Power, 2006; Raithel/Sarstedt/Scharf/Schwaiger, 2012, S. 509 ff.). Diese Felder können durch Zufriedenheitsstudien mit Bestandskunden aber allenfalls approximiert und somit in der Praxis nur unzureichend adressiert werden.

Selbstverständlich sind Kundenbefragungen wichtig und sollten insbesondere zur Messung der Produkt- und Servicequalität und zur Incentivierung kundennaher Bereiche unbedingt weiterhin herangezogen werden. Sie sind in der Praxis aber nicht annährend so relevant, wie es der Überhang an Bestandskunden- gegenüber Nicht-Kunden-Studien vermuten lässt. Für viele Unternehmen sind Kunden die wichtigste Ressource - sie sind jedoch nicht gleichzeitig die wichtigste Informationsquelle. Darum sollten Praktiker über eine Reallokation der Marktforschungsbudgets nachdenken. Sicherlich ist die Erreichbarkeit von Nicht-Kunden schwieriger und aufwändiger und die Stichprobenkosten höher. Der Mehrwert der Information wiegt dies je-

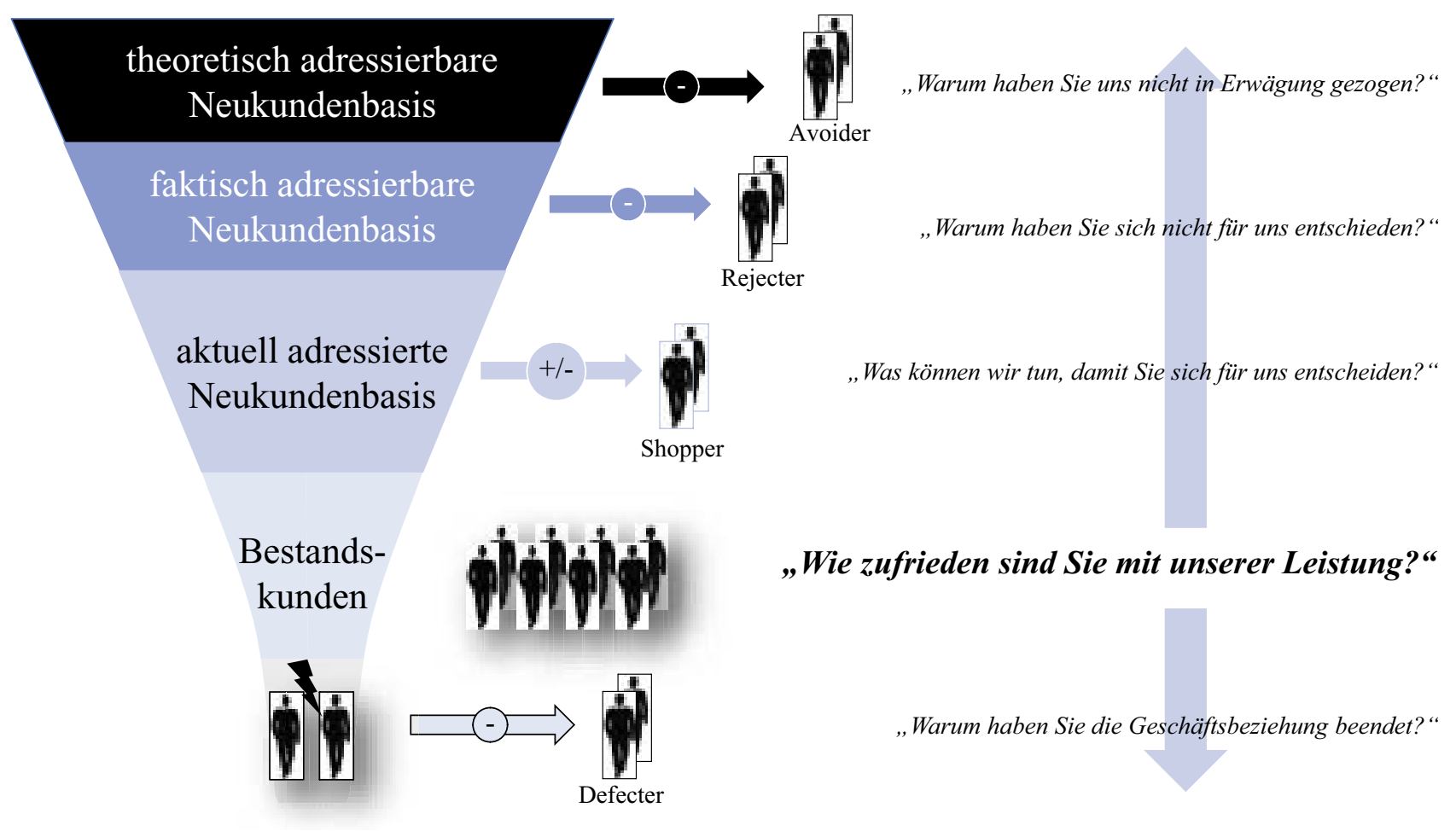

Abb. 1: Typen und Relevanz von Kunden und Nicht-Kunden 
doch in den meisten Fällen auf. Auch für wissenschaftliche Beiträge lohnt sich der Schritt zurück, um die Kundenforschung ganzheitlich zu betrachten und mehr als bisher ein weiteres Forschungsobjekt zum Thema zu machen: den Nicht-Kunden.

\section{Form des Forschungsdesigns}

Die Kritik an der zu häufigen Betrachtung des Bestandskunden als Objekt der Konsumentenforschung gilt in gleicher Form für großzahlige quantitative Studien als dominantes Element des Forschungsdesigns. Die Kundenforschung im Gastgewerbe, im Finanzdienstleistungssektor, in der Automobilbranche und in der Telekommunikation wird von Studien mit Likert-Skala dominierten Fragebögen beherrscht. Im akademischen Bereich hat sich in den letzten Jahren insbesondere bei Projekt- und Abschlussarbeiten von Studierenden die quantitative Onlinebefragung als Standard durchgesetzt. Auf der einen Seite ist dies durch die Verfügbarkeit von für Studierende kostenfreier und trotzdem leistungsfähiger Umfragesoftware wie z. B. Q-Set, Surveymonkey oder Rogator getrieben. Auf der anderen Seite vermittelt das quantitative Datenmaterial den Eindruck von Präzision und Eindeutigkeit und kann auch ohne dediziertes Methodenwissen mit deskriptiven Analysen beschrieben werden.

In vielen Fällen ist jedoch weder die Erhebungsmethode, noch die Form der Datenanalyse für die jeweils vorliegende Untersuchungsfrage optimal. Die Wahl der geeigneten Erhebungsmethode sollte stets aus dem Typ der vorliegenden Untersuchungsfrage abgeleitet und nicht ex ante definiert werden. Es lassen sich drei Formen unterscheiden: diffuse, mäßig definierte und klar definierte Untersuchungsfragen (vgl. Sarstedt/Mooi, 2014, S. 12 f., und Shiu/Hair/Bush/Ortinau, 2009).

Bei diffusen Untersuchungsfragen besteht Ungewissheit bzgl. der Maßnahmen und Hebel zur Lösung des Problems. Eine diffuse Untersuchungsfrage liegt typischerweise vor, wenn sich der Untersuchende einem neuen Problem erstmals nähert, erste Hypothesen generiert, oder ein neues Messinstrument für ein latentes Konstrukt wie $\mathrm{Zu}$ friedenheit, Loyalität, wahrgenommene Qualität oder Glück entwickelt. Es geht in dieser Phase einer Problembearbeitung darum, grundlegende Strukturen zu verstehen. Bei diffusen Untersuchungsfragen sind explorative Forschungsdesigns wie Beobachtungsstudien, Fokusgruppendiskussionen, Tiefeninterviews, Expertengespräche oder ethnografische Studien angebracht (vgl. Sarstedt/ Mooi, 2014, S. 15 f.). Vermieden werden sollten Designs, bei denen der Forscher bereits bei der Erstellung der Untersuchung einen hohen Strukturierungsgrad vorgeben muss, da er/sie zu diesem Zeitpunkt noch nicht über die notwendigen Erkenntnisse verfügt diese Strukturierung zu leisten. Eine unsachgemäße Struktur würde somit das Ergebnis der Untersuchung in unzulässigem Rahmen leiten und verfälschen. Fragebögen mit geschlossen formulierten Items sind somit tendenziell nicht angebracht.
Bei mäßig definierten Untersuchungsfragen sind zwar die Hebel zur Problemlösung grundsätzlich bekannt, es besteht jedoch Ungewissheit bzgl. der Ausgestaltung und des Zusammenspiels der Maßnahmen. Existiert für ein latentes Konstrukt ein Messmodell, welches nun konkret auf einen Sachverhalt angewendet werden soll, dann liegt eine mäßig definierte Untersuchungsfrage vor. Die Beschreibung eines Markts oder die Bildung von Marktsegmenten sind weitere Beispiele für mäßig definierte Problemstellungen. Diese verlangen nach einem deskriptiven Forschungsdesign. Auf maßgeblich quantitativen Fragebögen basierende Querund Längsschnittstudien sowie Paneluntersuchungen sind typische Beispiele (vgl. Sarstedt/Mooi, 2014, S. 17).

Klar definierte Untersuchungsfragen sind zu beantworten, wenn das Zusammenspiel der Maßnahmen und Hebel bekannt ist und das konkrete Ausmaß der Veränderung der Hebel auf die interessierende Größe gemessen werden soll. Darum sind bei solchen Fragenstellungen kausale Forschungsdesigns einzusetzen (vgl. Sarstedt/Mooi, 2014, S. 18 ff.). Von kausalen Designs wird verlangt, dass sie alternative Erklärungsmöglichkeiten für eine empirische gemessene Beziehung weitestgehend ausschließen (vgl. Atteslander, 2010). Darum sind Experimentalstudien in Form von Labor- oder Feldexperimenten sowie isolierte Testmärkte in diesem Kontext besonders geeignet. Allgemein gesprochen müssen die Designs ein hohes Maß an Kontrolle über die unabhängigen Variablen erlauben. Im Kontext der Konsumentenforschung zielen kausale Designs häufig auf die Messung der Reaktion des Kunden auf eine Änderung in dessen Entscheidungsumfeld. Hierbei kann es sich beispielsweise um Änderungen im Preisniveau, um neue Produkteigenschaften oder um veränderte Vertragsbedingungen handeln. Ziel ist die Simulation der Entscheidungssituation für den Probanden unter der Bedingung, dass nur die durch den Forscher bewusst induzierten Änderungen zur veränderten Verhaltensweise geführt haben. Der Einsatz eines mäßig definierten Erhebungsdesigns in Form eines Fragebogens würde in diesem Kontext in der Regel nicht zu validen Untersuchungsergebnissen führen.

Die direkte hypothetische Abfrage des eigenen zukünftigen Entscheidungsverhaltens liefert zum einen Antworten, die durch überlagernde Störfaktoren wie z. B. soziale Erwünschtheit verzerrt sind (vgl. Stocké, 2004, S. 303). Zum anderen werden Probanden durch diese Art der projektiven Befragung zu Prognosen des eigenen Verhaltens gedrängt, welches sie besonders bei komplexen und emotional aufgeladenen Produkten häufig nicht einmal ex post begründen können. Anders ausgedrückt ist die Vorhersagevalidität einer solchen Befragung bei Vorliegen einer klar definierten Untersuchungsfrage gering und sollte darum vermieden werden. Ebenso muss aber auch bei der Ausgestaltung eines kausalen Designs darauf geachtet werden, dass die Untersuchungssituation eine reale Entscheidung möglichst gut widerspiegelt, sofern das Ziel darin besteht, Aussagen über die Generalisierbarkeit eines Effekts in der Praxis zu treffen. Im Kontext von Kaufentscheidungen gehören hierzu beispielsweise die Einführung 
verbindlicher Kaufentscheidungen, das Angebot real existierender Produkte oder die Möglichkeit die Produkte vor dem Kauf zu begutachten (z. B. Lichters/Sarstedt/Vogt, 2015, S. 1 ff.).

Wie eingangs kritisiert, werden leider sowohl im unternehmerischen als auch im studentischen Umfeld zu häufig deskriptive Forschungsdesigns zur Beantwortung diffuser oder klar definierter Untersuchungsfragen eingesetzt. Dies geschieht in der Regel immer dann, wenn der Untersuchende sich ausschließlich auf die inhaltlichen Aspekte des vorliegenden Problems fokussiert und die zur empirischen Lösung der Problematik notwendigen methodischen Elemente außer Acht lässt. Vor dem Hintergrund des Umfelds dieser Personengruppen ist dies aber nicht verwunderlich. In Unternehmen sollen „Praktiker [..] Ergebnisse vorlegen, unabhängig von ihrem theoretischen Hintergrund." (Kleining, 2007, S. 189). Der Anreiz zu einer intensiven Auseinandersetzung mit den methodischen Grundlagen und Möglichkeiten des empirischen Designs ist somit begrenzt. Studierende wählen häufig deskriptive Erhebungsdesigns, da diese genau die Ergebnisse liefern können, mit denen sie sich auch in Lehrbüchern, der Tagespresse und in Praktikerzeitschriften konfrontiert sehen: Häufigkeitsangaben, Prozentsätze und relative Entwicklungen. Gleichzeitig bieten die eingangs des Abschnitts erwähnten Softwarelösungen eine professionelle Unterstützung bei der Datenerhebung und suggerieren eine fundierte Herangehensweise an die Problemstellung - auch wenn diese nicht notwendigerweise korrekt ist.

Gerade im Bereich der qualitativen Designs gibt es sowohl für Unternehmen als für Studierende viele interessante Ansätze zur Beantwortung von Problemstellungen mit geringem Strukturierungsgrad. In seiner populärwissenschaftlichen Monografie „Why We Buy: The Science of Shopping“ liefert Underhill mehrere praktische Beispiele von Unternehmen, die Beobachtungsstudien einsetzen um Erkenntnisse über die Verhaltensweisen der Besucher des Einzelhandels am Point of Sale zu erhalten (vgl. Underhill, 2008). Bemerkenswert an den Ergebnissen dieser Studien ist insbesondere, dass viele der Verhaltensweisen unbewusst ablaufen und sich die beobachteten Kunden weder an ihr Verhalten erinnern, noch dieses beschreiben oder gar begründen können. Die Ergebnisse einer direkten, fragebogengestützten Erhebung des Verhaltens in Form einer Kundenbefragung erfassen das faktische Verhalten somit nicht. Mit dem eigenen Verhalten konfrontierte Konsumenten können ihre Aktionen häufig nicht erklären, versuchen dies jedoch trotzdem. Kaiser (2007, S. 585) beschreibt dieses Verhalten als Post-Rationalisierung.

Interagiert der Forscher im Rahmen einer Beobachtungsstudie mit den Probanden und nimmt gemeinsam mit den Beobachteten an den interessierenden Abläufen teil, so wird dies als Ethnographie bezeichnet (vgl. Sarstedt/ Mooi 2014, S. 59). Procter \& Gamble, Whirlpool und die Citygroup sind Beispiele für Unternehmen, die Beobachter in die Haushalte ihrer (Nicht-)Kunden entsenden die dort mit diesen interagieren und bei der Informationssuche, der
Einkaufsplanung und der Verwendung der Produkte beobachten (vgl. Ohler/Samuel/McMurray, 2013).

Nachdem Volkswagen in den USA zwischen 2000 und 2005 einen Absatzeinbruch erlebt hatte, dem ein schlechtes Abschneiden in den wichtigen Kundenzufriedenheitsbarometern vorausgegangen war, entsandte die Unternehmensleitung ein Team aus Entwicklungsingenieuren und Marktforschern für eine ethnographische Studie mit dem Namen Moonraker in die USA. Mitglieder des Teams lebten zeitweise bei amerikanischen Familien, um deren Lebensgewohnheiten zu beobachten und US-amerikanische Reise-, Einkaufs- und Freizeitroutinen selbst zu erleben (vgl. Seith, 2006). Die so gewonnenen Erkenntnisse wurden später erfolgreich in Fahrzeugkonzepte umgesetzt. Bemerkenswert ist nicht nur, dass dieses Forschungsdesign Erkenntnisse lieferte, welche die bisher genutzten quantitativen Kundenzufriedenheitsstudien nicht hervorbrachten, sondern auch, dass sich die Akzeptanz der Forschungsergebnisse bezüglich der aus qualitativen Erhebung bereits bekannten und durch Ethnographie bestätigten Probleme unter den Fahrzeugentwicklern signifikant erhöhte. Das Resultat waren deutlich stringentere Lösungsansätze die in die neuen Ergebnisse einflossen. Dies ist ein grundlegender Vorteil qualitativer Studienergebnisse: sollen in Unternehmen Entscheidungen getroffen werden, so haben qualitative Kundenaussagen bei Entscheidern häufig eine höhere Überzeugungskraft als aggregierte Kundenfeedbacks auf Basis von statistischen Lagemaßen.

\section{Ausblick auf Teil 2}

Der zweite Teil dieses Beitrages wird sich mit den Kriterien zur Auswahl der geeigneten Datenanalysemethode befassen. Hier zeigt sich eine deutliche Diskrepanz zwischen Praktikern und Wissenschaftlern. In Unternehmen werden regelmäßig einfache Analyseverfahren der ersten Generation eingesetzt, die bei komplexen Problemstellungen nicht leistungsfähig genug sind. In der Wissenschaft kommen zu häufig hoch komplexe Methoden zur Lösung teilweise trivialer Probleme zum Einsatz. Kundenforscher, egal ob in Wissenschaft oder Praxis, müssen sich bewusst machen, dass der Einsatz von Datenanalyseverfahren keinem Selbstzweck dient. Vielmehr gilt es, eine Balance zwischen methodischem Anspruch einerseits und der häufig gebotenen Einfachheit andererseits zu finden.

\section{Literatur}

ACSI, History of the American Customer Satisfaction Index, 2015. Online im Internet: URL: http://www.theacsi.org/aboutacsi/history (Abrufdatum 06.07.2015).

Atteslander, P., Methoden der empirischen Sozialforschung, 13. Aufl., Berlin 2010.

Denove, C., J. D. Power, Satisfaction - How Every Great Company Listens to the Voice of the Customer, New York 2006.

Die Welt, Sonderausgabe: Service Champions vom 16.10.2014. Online im Internet: http://www.servicevalue.de/fileadmin/contents/04_Wettbewerbe/Service-Champions/pdf/Service-Champions_Sonderthema_DIE_WELT_16.10.2014.pdf (Abrufdatum 06.07.2015). 
Hauser, J. R., D. I. Simester, B. Wernerfelt, Customer Satisfaction Incentives, in: Marketing Science, Vol. 13 (1994), Nr. 4, S. 327-350.

J. D. Power, J. D. Power und AUTO TEST melden: Kaufentscheidungen von Neuwagenkäufern in Deutschland werden maßgeblich von Zuverlässigkeit, Langlebigkeit und Fahrsicherheit beeinflusst, 29.05.2014, Pressemitteilung.

Jobber, D., Principles and Practice of Marketing, 7. Aufl., New York 2012.

JDPA, Automotive Studies, 2014. Online im Internet: URL: http://ratings.jdpower.com/automotive/index.htm (Abrufdatum 10.04.2015).

Kaiser, W., Fast Moving Consumer Goods - Zwischen Artefakt und Consumer Insight, in: Naderer, G., Balzer, E. (Hrsg.), Qualitative Forschung in Theorie und Praxis - Grundlagen Methoden und Anwendungen, Wiesbaden 2007, S. 585-593.

Kleining, G., Der qualitative Forschungsprozess, in: G. Naderer, E. Balzer (Hrsg.), Qualitative Forschung in Theorie und Praxis - Grundlagen Methoden und Anwendungen, Wiesbaden, 2007, S. 189-230.

Lichters, M., M. Sarstedt, B. Vogt, On the Practical Relevance of the Attraction Effect: A Cautionary Note and Guidelines for Context Effect Experiments, in: AMS Review, Vol. 5 (2015), Nr. 1-2, S. 1-19.

Nieschlag, R., E. Dichtl, H. Hörschgen, Marketing, Auflage: 19. Aufl., Berlin 2002.

Ohler, M., P. Samuel, M. McMurray, Ethnography: Is Your Company Missing the Train?, 2013. Online im Internet: URL: http://www.industryweek.com/innovation/ethnography-yourcompany-missing-train (Abrufdatum, 01.07.2015).

Raithel, S., M. Sarstedt, S. Scharf, S., M. Schwaiger, On the Value Relevance of Customer Satisfaction. Multiple Drivers and Multiple Markets, in: Journal of the Academy of Marketing Science, Vol. 40 (2012), Nr. 4, S. 509-525.

Rettie, R., C. Brewer, The Verbal and Visual Components of Package Design, in: Journal of Product \& Brand Management, Vol. 9 (2000), Nr. 1 S. 56-70.

Sarstedt, M., E. A. Mooi, A Concise Guide to Market Research: The Process, Data, and Methods Using IBM SPSS Statistics, 2. Aufl., Heidelberg et al. 2014.

Schütz, T., K. Kindermann, Fragen Sie Ihre Nicht-Kunden - Potentielle Kunden liefern die wertvollsten Informationen, in: Prokom Report, Nr. 03 (2012), S. 102-103.

Sharma, A., D. Sarel, The Impact of Customer Satisfaction Based Incentive Systems on Salespeople's Customer Service Response: An Empirical Study, in: Journal of Personal Selling \& Sales Management, Vol. 15, No. 3 (1995), S. 17-29.

Shiu, E., J. Hair, R. Bush, D. Ortinau, Marketing Research, London 2009.

Seith, A., Codename „Moonraker“: VW erforscht den American Way of Life, in: Spiegel Online. Online im Internet: URL: http://www.spiegel.de/wirtschaft/codename-moonraker-vw-erforscht-den-american-way-of-life-a-394873.html (Abrufdatum: 01.07.2015).

Stocké, V., Entstehungsbedingungen von Antwortverzerrungen durch soziale Erwünschtheit, in: Zeitschrift für Soziologie, Vol. 33 (2004), Nr. 4, S. 303-320.

Underhill, P., Why We Buy: The Science of Shopping, New York 2008.

Uttl, B., Measurement of Individual Differences: Lessons from Memory Assessment in Research and Clinical Practice, in: Psychological Science, Vol. 16 (2005), Nr. 6, S. 460-467.

\section{International aufgestellt.}

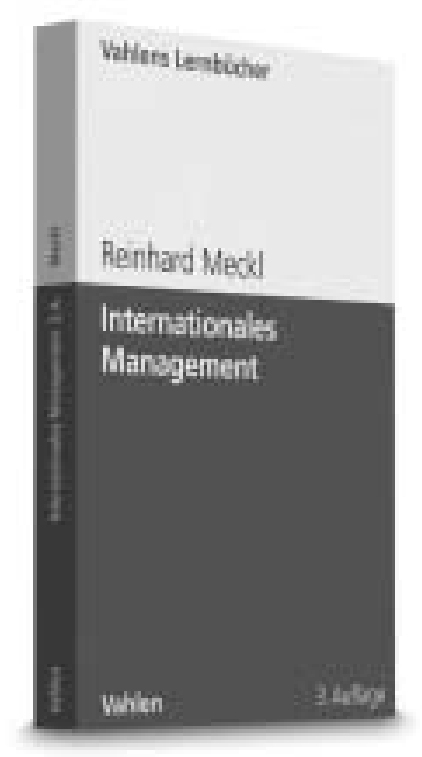

Meckl, Internationales Management

3. Auflage. 2014. XXVI, 354 Seiten. Kartoniert $€ 29,80$ ISBN 978-3-8006-4784-2

Portofrei geliefert: vahlen.de/13174179

\section{Das Lehrbuch}

vermittelt anschaulich die theoretischen Grundlagen des Internationalen Managements, um ausländische Märkte erfolgreich bearbeiten zu können. Neben den wichtigsten Erklärungsansätzen, Führungsmodellen und Managementmethoden werden auch die wesentlichen Funktionsfelder eines internationalen Unternehmens sowie das Management von speziellen Risiken dargestellt.

\section{Schafft Wissensvorsprung}

für Studenten der Wirtschaftswissenschaften an Universitäten, Fachhochschulen und Berufsakademien.

Erhältlich im Buchhandel oder bei: vahlen.de | Verlag Franz Vahlen GmbH 80791 München | bestellung@vahlen.de | Preise inkl. MwSt.

\section{Vahlen}

